\title{
Argemone mexicana decoction for the treatment of uncomplicated falciparum malaria
}

\author{
Merlin L. Willcox ${ }^{a, b, *}$, Bertrand Graz ${ }^{a}$, Jacques Falquet ${ }^{a}$, Oumar Sidibé ${ }^{c}$, \\ Mathieu Forster ${ }^{d, e}$, Drissa Diallo ${ }^{c}$
}

\author{
a Antenna Technologies, Geneva, Switzerland \\ ${ }^{\mathrm{b}}$ Research Initiative for Traditional Antimalarial Methods (RITAM), Buckingham, UK \\ c Département de Médecine Traditionnelle, INRSP, Bamako, Mali \\ ${ }^{d}$ Faculté de Médecine, Université Laval, Canada \\ e Institute of Social \& Preventive Medicine, University of Bern, Switzerland
}

Received 14 June 2006; received in revised form 21 May 2007; accepted 22 May 2007

\author{
KEYWORDS \\ Uncomplicated \\ malaria; \\ Plasmodium \\ falciparum; \\ Argemone mexicana; \\ Traditional medicine; \\ Clinical trial; \\ Mali
}

\begin{abstract}
Summary A prospective, dose-escalating, quasi-experimental clinical trial was conducted with a traditional healer using a decoction of Argemone mexicana for the treatment of malaria in Mali. The remedy was prescribed in three regimens: once daily for 3 days (Group A; $n=23$ ); twice daily for 7 days (Group B; $n=40$ ); and four times daily for the first 4 days followed by twice daily for 3 days (Group C; $n=17$ ). Thus, 80 patients were included, of whom $80 \%$ were aged $<5$ years and $25 \%$ were aged $<1$ year. All presented to the traditional healer with symptoms of malaria and had a Plasmodium falciparum parasitaemia $>2000 / \mu$ l but no signs of severe malaria. The proportions of adequate clinical response (ACR) at Day 14 were $35 \%, 73 \%$ and $65 \%$ in Groups A, B and C, respectively $(P=0.011)$. At Day 14 , overall proportions of ACR were lower in children aged $<1$ year $(45 \%)$ and higher in patients aged $>5$ years $(81 \%)(P=0.027)$. Very few patients had complete parasite clearance, but at Day 14, 67\% of patients with ACR had a parasitaemia $<2000 / \mu \mathrm{l}$. No patient needed referral for severe disease. Only minor side effects were observed. Further research should determine whether this local resource could represent a first-aid home treatment in remote areas.

(c) 2007 Royal Society of Tropical Medicine and Hygiene. Published by Elsevier Ltd. All rights reserved.
\end{abstract}

\section{Introduction}

Malaria mortality and morbidity is increasing in Africa despite initiatives to control it (Anonymous, 2005). Many patients rely on traditional herbal medicines as their first line of treatment, especially in rural areas. However, few clinical studies have been conducted to evaluate their safety and efficacy.
* Corresponding author. Present address: 66 Lye Valley, Oxford OX3 7ER, UK. Mobile: +44 7910329164.

E-mail address: merlinwillcox@doctors.org.uk (M.L. Willcox).

0035-9203/\$ - see front matter @ 2007 Royal Society of Tropical Medicine and Hygiene. Published by Elsevier Ltd. All rights reserved. doi:10.1016/j.trstmh.2007.05.017 
In Mali, the Department for Traditional Medicine, within the Ministry of Health, produces standardised 'improved traditional medicines' (médicaments traditionnels améliorés (MTA)), which are included in the National Formulary (Ministère de la Santé, des Personnes Agées et de la Solidarité, 1998). Currently, a mixture of three herbs called 'Malarial' is marketed as an antimalarial (Diallo et al., 2004) but it has never been tested in children under the age of 5 years. This study was undertaken to provide data that could support the development of a new MTA as well as official recommendations on the use of local resources in situations where standard modern drugs are lacking despite all possible efforts.

An ethnobotanical and retrospective treatmentoutcome clinical study in the Sikasso and Bandiagara regions of Mali led to the recognition of the widespread use of plants by local communities in the management of malaria and identified a number of plants used (Diallo et al., 2006). Patients were asked about their recent experience of clinical outcomes following the use of diverse treatments for malaria, and the plants with the best outcomes were also tested in vitro at the Swiss Tropical Institute in Basel. The most effective plant, according both to the patients and to the in vitro tests, was Argemone mexicana $\mathrm{L}$ (Papaveraceae). The $50 \%$ inhibitory concentration $\left(\mathrm{IC}_{50}\right)$ values of the aerial parts against the chloroquine-resistant K1 strain of Plasmodium falciparum were 5.89, 6.22, 1.22 and $1.00 \mu \mathrm{g} / \mathrm{ml}$ for the aqueous decoction, aqueous maceration, and dichloromethane and methanol extracts, respectively.

Argemone mexicana is a pantropical weed originating from Mexico (Figure 1). It has a long history of use in traditional medicine, dating back to the Aztecs (Emmart, 1940). After the plant spread to India, it was used to treat a wide variety of diseases in Ayurvedic and Unani medicine, including malaria (Nadkarni, 1976). It is also used as an antimalarial in several African countries, including Benin, Mali and Sudan. Its in vitro efficacy against $P$. falciparum has been confirmed by several studies (Adjobimey et al., 2004; Diallo et al., 2006; Simonsen et al., 2001). No previous clinical studies have been conducted to determine its safety and efficacy in human patients.

To our knowledge, this study is unique in herbal antimalarial research in its focus on young children in subSaharan Africa, the most vulnerable group.

\section{Materials and methods}

\subsection{Setting}

This study was conducted in the village of Missidougou, in the Sikasso region (southwest) of Mali. The village is located $40 \mathrm{~km}$ from the nearest Malian primary health centre and $70 \mathrm{~km}$ from the nearest hospital, including a $25 \mathrm{~km}$ dirt track along which there is no public transport.

The village chief of Missidougou is a traditional healer (Figure 1). His grandfather taught him to treat malaria using A. mexicana and he has already passed this knowledge to his son. There is therefore the experience of at least four generations in the use of this plant for the treatment of malaria in Missidougou. A previous ethnomedical survey found that use

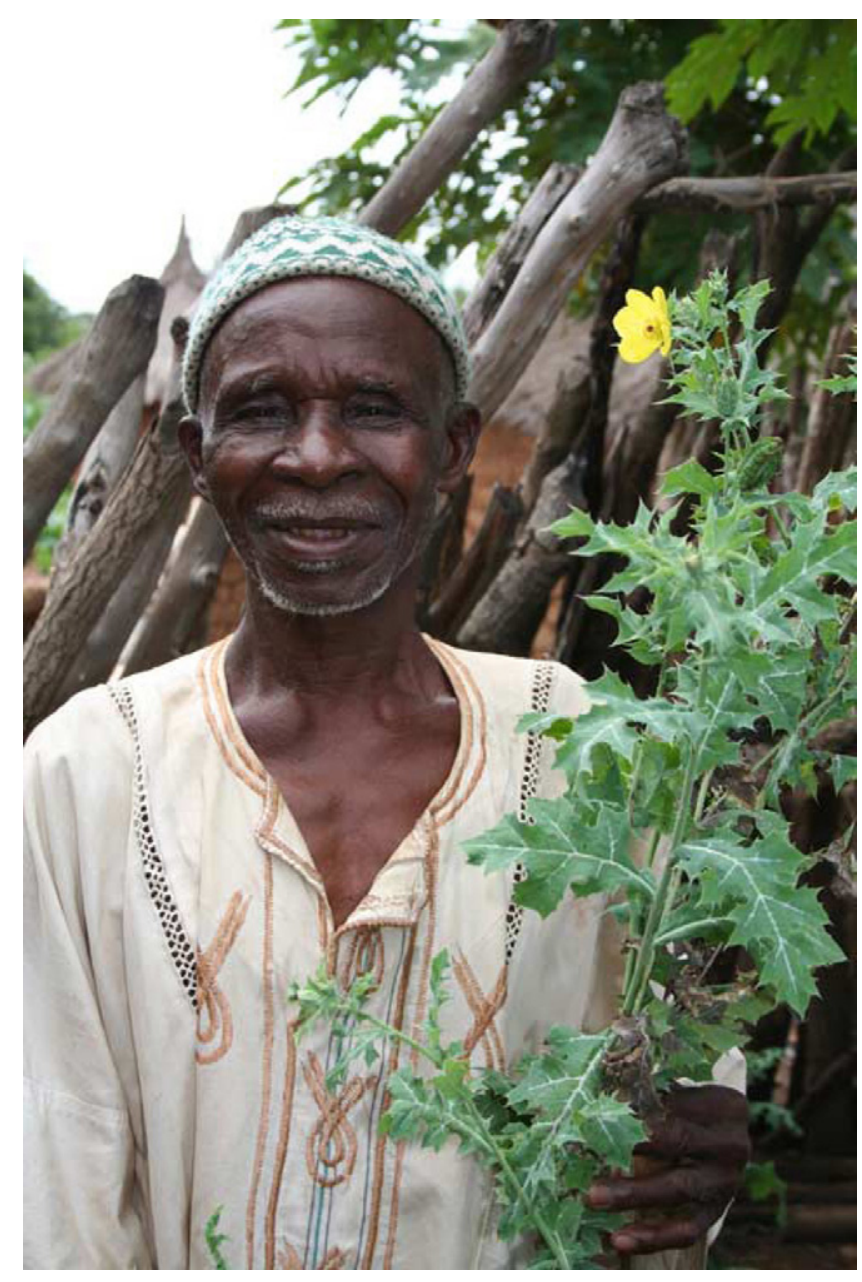

Figure 1 Chief Tiemoko Bengaly holding Argemone mexicana, the plant his grandfather taught him to use as a treatment for malaria. ${ }^{\circ}$ Merlin L. Willcox.

of this treatment was systematically associated with good outcomes (Diallo et al., 2006; Graz et al., 2005).

\subsection{Participants}

Patients consulting the traditional healer and diagnosed with malaria by him were sent for blood films and clinical evaluation. Those with a pure $P$. falciparum parasitaemia $>2000 / \mu \mathrm{l}$, with symptoms of malaria within the last $24 \mathrm{~h}$ and without any signs of severe disease were asked for their consent to participate. There was an information sheet, which patients were given to keep for reference and which was read and translated to the patients and their parents in the local language, as most were illiterate. Subjects gave individual written consent, usually with a fingerprint as most could not sign their name.

Exclusion criteria included: severe malaria; pregnancy (although subsequent enquiry revealed that $A$. mexicana is administered to pregnant women, with no reported problems); age <3 months; other concomitant febrile illness; administration of a full course of antimalarial (modern or traditional) within the previous week; lack of consent; and inability to return for follow-up. 
Those excluded from the study received traditional treatment. Patients whom the traditional healer could not treat (including those with severe malaria) were referred to the medical team for a consultation, and treated with modern medicine if appropriate.

\subsection{Preparation and dosage}

Argemone mexicana grows like a weed in villages of the Sikasso region of Mali. The aerial parts are harvested and dried in the shade. The antimalarial decoction is prepared by filling a cooking pot with as much dried plant material as possible (approximately $1 \mathrm{~kg}$ ), filling it with water (approximately $10 \mathrm{l}$ ) and boiling it for $3 \mathrm{~h}$. The decoction is drunk hot or allowed to cool. It can be kept in a closed container for a few days. Often the traditional healer also washes the patient (especially small children) with the decoction and the mother sometimes gives the preparation intrarectally to toddlers, but in the context of this study these routes of administration were not investigated.

The traditional healer determined the unit dose of the decoction, which varied in quantity according to the age of the patient. The healer prescribed a single dose daily for 3 days ('lower range' regimen) to the first 23 patients (Group A). The following cohort of 40 patients (Group B) received one dose twice a day for 7 days (the average usual regimen). A final group of 17 patients (Group C) were then prescribed the 'upper range' regimen: one dose four times a day for the first 4 days, then one dose twice daily until the eighth day.

It is important to emphasise that the dose escalation was not planned as part of the study design. The traditional healer was asked to determine the dose and at first decided to prescribe dose ' $A$ '. When it became apparent that this was not very effective, the team questioned him and he explained he thought that this dose was more 'scientific' (perhaps because it is the same dosage frequency as chloroquine). The team asked what the usual dose was and he replied that he normally gives the dried plant to the patients to prepare at home and told them to drink as much as possible. There had been no standard frequency or duration of treatment. The healer and the research team mutually agreed on dosage schedules ' $B$ ' and ' $C$ ' as a standardisation of average and 'upper range' doses.

\subsection{Design}

The study was a prospective, dose-escalating, quasiexperimental (since no randomisation was performed to allocate participants to the treatment groups) clinical trial. Patients were seen on Days $0,1,2,3,7,14$ and 28, and any intermediate day if they became unwell. On each visit, patients were assessed clinically. Blood films were taken on all visits except Day 1. A sample of venous blood was taken on Days 0, 3, 7 and 14 for measurement of haematocrit, white cell count, platelet count, liver enzymes (aspartate aminotransferase (AST) and alanine aminotransferase (ALT)) and creatinine. Haematocrit was also measured on Day 28. Electrocardiograms (ECG) were taken on Days 0, 3, 7 and 14.
In cases of treatment failure, patients were given alternative treatment with standard first-line antimalarials (which were chloroquine or sulfadoxine/pyrimethamine at that time). No further blood tests were taken after commencement of the alternative treatment, unless clinically indicated.

\subsection{Main outcome measures}

The outcome measures were those defined by the Research Initiative for Traditional Antimalarial Methods guidelines for studies on traditional antimalarial remedies (Willcox and Olanrewaju, 2004). These are based on WHO (1996) definitions. For this study, 'fever' was defined as history of fever in the previous $24 \mathrm{~h}$, or axillary temperature $\geq 37.5^{\circ} \mathrm{C}$.

Adequate clinical response (ACR) was defined as the absence of parasitaemia on Day 14 irrespective of temperature or symptoms, or absence of fever irrespective of the presence of parasitaemia, without previously meeting any of the criteria of early or late treatment failure.

Early treatment failure (ETF) was defined as development of danger signs on Days 1, 2 or 3 in the presence of parasitaemia; fever on Day 2 with parasitaemia greater than at baseline; or fever on Day 3 with parasitaemia. (Afebrile patients with parasitaemia on Day $3 \geq 25 \%$ of the count on Day 0 were not counted as ETF but were observed closely.)

Late treatment failure was defined as development of any danger signs or signs of severe malaria in the presence of parasitaemia on any day from Days 4 to 14, without previously meeting any of the criteria of ETF; or fever with parasitaemia on any day from Days 4 to 14 , without previously meeting any of the criteria of ETF.

\subsection{Laboratory methods}

A field laboratory was installed in one of the village houses, using a solar panel and car battery to power microscopes, centrifuges and an ECG machine. Blood films were stained with $10 \%$ Giemsa for $10 \mathrm{~min}$, and parasite counts were done using standard WHO criteria. All blood films were checked by two independent microscopists. If there was a difference $>100 \%$ between the two results, it was repeated by a third microscopist until there were two concordant results.

Haematocrit was measured using microhaematocrit tubes in a centrifuge. White blood cells and platelets were counted manually using a Malassez chamber. Glucose was measured on Day 0 using a glucometer (Ascensia Breeze; Bayer HealthCare, Newbury, UK). AST, ALT and creatinine were measured by the laboratory at Sikasso Hospital. Blood was centrifuged within a few hours of the sample being taken and the serum was transported to the hospital within $72 \mathrm{~h}$. ECGs were taken using a Cardisuny 501 SA single channel electrocardiograph (Fukuda M-E Kogyo Co. Ltd., Tokyo, Japan) and analysed manually. ECGs and venous blood samples were not routinely taken from children aged $<5$ years. Blood was also taken on filter paper at inclusion and was stored. Patients who had a negative blood film at Day 14 but parasitaemia again by Day 28 had a second filter-paper blood sample taken for PCR analysis to determine whether these patients had a recrudescence or a re-infection. 


\subsection{Statistical analysis}

Data were entered and analysed using Epi Info 5.01 (CDC, Atlanta, GA, USA) and SPSS 11.0 (SPSS Inc., Chicago, IL, USA) software. Comparison of baseline characteristics of included patients was performed using Pearson's $\chi^{2}$, Fisher's exact test, one-way ANOVA and Kruskal-Wallis non-parametric ANOVA as appropriate. Comparison of group-specific results was assessed using the $\chi^{2}$ test for linear trend.

The univariate odds ratio for ACR was calculated with $95 \%$ $\mathrm{Cl}$. Subsequently, a multiple logistic regression analysis was performed, including variables with a level of significance of $P<0.25$ in the univariate analysis. Haematocrit, leukocyte and platelet counts over time were assessed with a multilevel regression model for repeated measures (Raudenbush, 2001; Raudenbush and Bryk, 2002). The software used was Hierarchical Linear and Non Linear Modelling (HLM), version 5.0 (Raudenbush et al., 2001).

\section{Results}

\subsection{Patients included}

From mid September to mid November 2004, which is the peak malaria season in the region, 251 patients were diagnosed with 'soumaya' (malaria) by the traditional healer and were screened for inclusion in this study. Of these, 167 patients were excluded, with the principal reason being insufficient parasitaemia $(n=118)$. Other reasons were absence of parasites $(n=30)$, concomitant illness $(n=5)$ and miscellaneous $(n=14)$.

Eighty-four patients were initially included, but three of these were subsequently excluded because of withdrawal of consent $(n=2)$ or discovery of an exclusion criterion $(n=1)$. One patient did not fall into any of the three main dosage groups, so was excluded from the main analysis.

\subsection{Baseline characteristics}

The three dosage groups are comparable in most respects (see Table 1). However, splenomegaly was significantly more frequent in Group A than in Groups B and C, the initial tem-
Table 2 Total doses taken by patients over the course of treatment $(\mathrm{ml} / \mathrm{kg})$

\begin{tabular}{lccc}
\hline & Group A & Group B & Group C \\
\hline Mean total dose & 12.9 & 84.6 & 152.0 \\
Minimum total dose & 6.5 & 28.1 & 23.8 \\
Maximum total dose & 25.7 & 162.0 & 333.0 \\
\hline
\end{tabular}

perature in Group A was higher than in Groups B and C and the initial parasitaemia was lower in Group $B$ than in Groups $A$ and $C$. This could be explained by the fact that at the start of the study, many patients came to be screened (exceeding the capacity of the research team), therefore it was necessary to prioritise the patients who had higher measured temperatures. Very few patients (one in Group A, two in Group B and none in Group C) had taken any antimalarials in the preceding week.

\subsection{Dosage and compliance}

Although not every administration of medicine was observed, patients were asked about their compliance with the prescribed treatment. The reported dose was calculated for each patient and summarised as a total dose over the course of treatment (Table 2). There was no overlap between Groups A and B, and the dose taken by Group B was on average 6.5 times greater than the dose taken by Group A. There was not such a large increase in dose between Groups $B$ and $C$.

Reported compliance was better in Group A than in Groups B or C; $40 \%$ of patients in Group B and 35\% of those in Group $C$ took less than the dose prescribed, but approximately $50 \%$ of patients in both groups took more than the dose prescribed.

\subsection{Clinical efficacy}

Overall results at Day 14 by intention-to-treat are shown in Table 3. There is a statistically significant difference between Group A (35\% ACR) and Group B (73\% ACR). Groups

Table 1 Baseline characteristics of patients included in the study

\begin{tabular}{|c|c|c|c|c|}
\hline & Group A $(n=23)$ & Group B $(n=40)$ & Group C $(n=17)$ & $P$-value \\
\hline No. (\%) male & $12(52 \%)$ & $20(50 \%)$ & $9(53 \%)$ & $0.974^{\mathrm{a}}$ \\
\hline Median age (years) & 2.29 & 1.92 & 2.00 & $0.944^{\mathrm{b}}$ \\
\hline Median weight:age ratio (kg/years) & 5.0 & 5.5 & 4.75 & $0.656^{\mathrm{b}}$ \\
\hline Splenomegaly present (\%) & $13(57 \%)$ & $11(28 \%)$ & $3(18 \%)$ & $0.005^{\mathrm{a}}$ \\
\hline Hepatomegaly present (\%) & $3(13 \%)$ & $4(10 \%)$ & $1(6 \%)$ & $0.115^{\mathrm{a}}$ \\
\hline Mean temperature $\left({ }^{\circ} \mathrm{C}\right)( \pm \mathrm{SD})$ & $38.4( \pm 0.7)$ & $37.8( \pm 1.0)$ & $37.8( \pm 0.7)$ & $0.024^{c}$ \\
\hline Geometric mean parasite count (per $\mu \mathrm{l}$ ) & 19634 & 14825 & 33884 & $0.050^{c}$ \\
\hline Haematocrit $(\%)( \pm S D)^{d}$ & $32.7( \pm 5.2)[18]$ & $30.6( \pm 6.5)[35]$ & $27.8( \pm 5.3)[17]$ & $0.056^{c}$ \\
\hline Mean glucose $(\mathrm{mmol} / \mathrm{l})( \pm \mathrm{SD})^{\mathrm{d}}$ & $5.7( \pm 1.5)[19]$ & $5.6( \pm 1.3)[33]$ & $6.3( \pm 1.4)[9]$ & $0.404^{c}$ \\
\hline
\end{tabular}

a Pearson's $\chi^{2}$ or Fisher's exact test.

b Kruskal-Wallis test.

c One-factor ANOVA.

${ }^{\mathrm{d}}$ Number of patients tested in square brackets. 
Table 3 Overall results at Days 14/28 according to treatment group (\%)

\begin{tabular}{lcccc}
\hline & Group A $(n=23)$ & Group B $(n=40)$ & Group C $(n=17)$ & $P$-value \\
\hline Adequate clinical response & $35 / 22$ & $73 / 63$ & $65 / 59$ & $0.011 / 0.027$ \\
Early treatment failure & $26 / 26$ & $15 / 15$ & $12 / 12$ & 0.420 \\
Late treatment failure & $35 / 39$ & $13 / 23$ & $24 / 29$ & $0.111 / 0.372$ \\
Lost to follow-up & $9 / 9$ & $0 / 0$ & $0 / 0$ & 0.079 \\
\hline
\end{tabular}

${ }^{a} \chi^{2}$ test for linear trend.

Table 4 Percentage of patients with adequate clinical response at Days 14/28, according to age group ${ }^{a}$

\begin{tabular}{|c|c|c|c|c|c|c|}
\hline Age (years) & Group A $(n=23)$ & Group B $(n=40)$ & Group C $(n=17)$ & $P$-value ${ }^{\mathrm{b}}$ & Totalc & $P$-value ${ }^{\mathrm{b}}$ \\
\hline$<1(n=20)$ & $29 / 0[7]$ & $60 / 50[10]$ & $33 / 33[3]$ & N/A & $45 / 30$ & $0.027 / 0.12$ \\
\hline $1-5(n=44)$ & $25 / 17[12]$ & $71 / 62[21]$ & $73 / 64[11]$ & $0.019 / 0.079$ & $59 / 50$ & \\
\hline$>5(n=16)$ & $75 / 75[4]$ & $89 / 78$ [9] & $67 / 67[3]$ & N/A & $81 / 75$ & \\
\hline
\end{tabular}

a Figures in square brackets indicate the total number of patients in each group.

${ }^{b} \chi^{2}$ test for linear trend (Groups $<1$ year and $>5$ years not tested).

c Groups A, B and C pooled together.

$B$ and $C$ had similar results ( $73 \%$ vs. $65 \%$ ACR). These results can be further broken down by age group (see Table 4). Within the age group $1-5$ years, there was a very significant difference in the proportion of ACRs in dose Group A (25\%) compared with dose Group B (71\%). Age- and group-specific significant differences remain even when adjusting for differences in baseline characteristics, as assessed by a logistic regression model (see Table 5). Of the patients with ACR at Day $14,56 \%, 86 \%$ and $91 \%$ in Groups A, B and C, respectively, still had an ACR by Day 28 .

The age groups $<1$ year and $>5$ years were too small to be able to detect any differences within these groups according to the dosage. However, patients aged $<1$ year were almost half as likely to have an ACR compared with

Table 5 Univariate and multivariate analyses of adequate clinical response at day 14

\begin{tabular}{|c|c|c|c|c|}
\hline \multirow[t]{2}{*}{ Variable } & \multicolumn{2}{|c|}{ Univariate analysis } & \multicolumn{2}{|c|}{ Multiple logistic regression } \\
\hline & OR $(95 \% \mathrm{Cl})$ & $P$-value & AOR $(95 \% \mathrm{CI})$ & $P$-value \\
\hline \multicolumn{5}{|l|}{ Treatment group } \\
\hline A & 1 (reference) & & 1 (reference) & \\
\hline B & $4.9(1.6-14.9)$ & 0.005 & $4.5(1.3-15.2)$ & 0.016 \\
\hline $\mathrm{C}$ & $3.4(0.9-12.8)$ & 0.065 & $3.1(0.7-13.1)$ & 0.130 \\
\hline \multicolumn{5}{|l|}{ Age group (years) } \\
\hline$<1$ & 1 (reference) & & 1 (reference) & \\
\hline $1-5$ & $1.9(0.7-5.5)$ & 0.222 & $1.7(0.5-5.5)$ & 0.366 \\
\hline$>5$ & $5.8(1.3-26.5)$ & 0.024 & $5.1(1.0-26.4)$ & 0.055 \\
\hline \multicolumn{5}{|c|}{ Baseline temperature $\left({ }^{\circ} \mathrm{C}\right)$} \\
\hline$\geq 38.5$ & 1 (reference) & & 1 (reference) & \\
\hline $38.4-37.5$ & $1.1(0.4-3.0)$ & 0.899 & $1.0(0.3-3.1)$ & 0.981 \\
\hline$<37.5$ & $3.4(0.9-13.6)$ & 0.078 & $1.5(0.3-7.2)$ & 0.604 \\
\hline \multicolumn{5}{|c|}{ Baseline parasite count (per $\mu l$ ) } \\
\hline$\geq 30000$ & 1 (reference) & & & \\
\hline $29999-10000$ & $1.3(0.4-4.1)$ & 0.610 & & \\
\hline$<10000$ & $1.1(0.4-3.3)$ & 0.546 & & \\
\hline \multicolumn{5}{|l|}{ Splenomegaly } \\
\hline Yes & 1 (reference) & & & \\
\hline No & $1.2(0.5-3.0)$ & 0.753 & & \\
\hline \multicolumn{5}{|l|}{ Hepatomegaly } \\
\hline Yes & 1 (reference) & & & \\
\hline No & $0.9(0.2-3.9)$ & 0.855 & & \\
\hline
\end{tabular}

OR: odds ratio; AOR: adjusted odds ratio. 


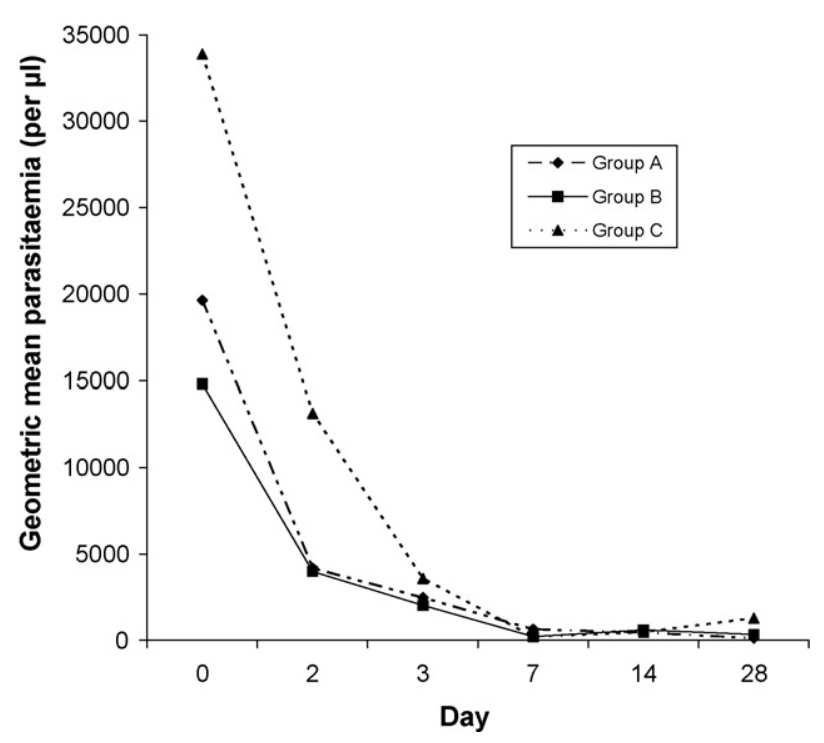

Figure 2 Parasitaemia in all patients during treatment with Argemone mexicana.

those aged $>5$ years $(45 \%$ vs. $81 \% ; P=0.027)$ (Table 4$)$. Overall ACR gave comparable results when adjusted for age and baseline temperature. Baseline parasite count, splenomegaly and hepatomegaly were not significantly associated with ACR at day 14 (Table 5).

Of the patients with treatment failure, only one showed some signs compatible with severe malaria: a history of repeated convulsions and hyperparasitaemia. The patient was conscious and able to drink when reviewed, so was treated with oral artemether/lumefantrine $\left(\right.$ Coartem $\left.^{\circledR}\right)$ and made a good, uncomplicated recovery. All the other treatment failures were patients with persistent fever and parasitaemia despite treatment, but had no danger signs.

\subsection{Parasitological efficacy}

Figure 2 shows the change in parasitaemia in the course of treatment in all patients (parasitaemia was no longer measured after commencement of a conventional antimalarial). The majority of patients with ACR at Day 14 still had a measurable parasitaemia, albeit at a lower level than at the start of treatment. Of these patients, the parasitaemia was $<2000 / \mu$ l (the minimum parasitaemia needed for inclusion in this study) in $88 \%$ of those in Group A, 59\% in Group B and $73 \%$ in Group C (overall 67\%). Only four patients in Group B $(14 \%$ of ACRs) and three in Group C (27\% of ACRs) had complete parasite clearance at Day 14. Of these, five had measurable parasitaemia again at Day 28. PCR was only obtainable for one of these patients and suggested a recrudescence.

\subsection{Safety}

Several studies have documented the toxicity of the oil obtained from $A$. mexicana seeds. These are similar in appearance to mustard seeds and sometimes their oil has been found as a contaminant in mustard oil used for cooking, resulting in 'epidemic dropsy' in those inadvertently consuming it (Tandon et al., 1975). The toxic constituent was shown to be the alkaloid sanguinarine (Sarkar, 1948). However, using HPLC and mass spectrometry analysis we were unable to detect sanguinarine in the herbal tea prepared from $A$. mexicana in Mali. Experiments have proven that sanguinarine is nearly exclusively located in the seeds of A. mexicana (A. Diop, K. Hostettmann's group, University of Geneva, Switzerland, personal communication). Even pure crushed seeds boiled for $3 \mathrm{~h}$ in water produced an aqueous phase devoid of sanguinarine, probably because this compound is destroyed by such treatment or because it is not extracted by water under these conditions.

Acute toxicity testing of this tea in mice showed no evidence of any adverse effects at doses up to $3.2 \mathrm{~g} / \mathrm{kg}$ of the freeze-dried tea, equivalent to $35 \mathrm{~g} / \mathrm{kg}$ of the plant powder (Sidibe, 2006).

Patients were asked about any new symptoms they were experiencing and these were assumed to be adverse effects of the medication, although it is possible that they had other causes. Cough and diarrhoea were the commonest 'adverse events' and were reported in 17-25\% of patients in each group. All of these were graded as mild or moderate. There were no severe adverse events, and no patient had to stop treatment because of an adverse event. These adverse events were not correlated with a higher dose of treatment.

Among available blood tests, multilevel regression models showed an overall significant increase in haematocrit between Day 0 and Day 28 in Group C $(P=0.001)$ but not in Groups A $(P=0.570)$ or $\mathrm{B}(P=0.069)$ (Figure 3$)$. No significant difference was observed in the progression of white cell counts. The platelet counts increased significantly between Days 0 and 14 in Groups B $(P=0.043)$ and $C(P<0.001)$; no
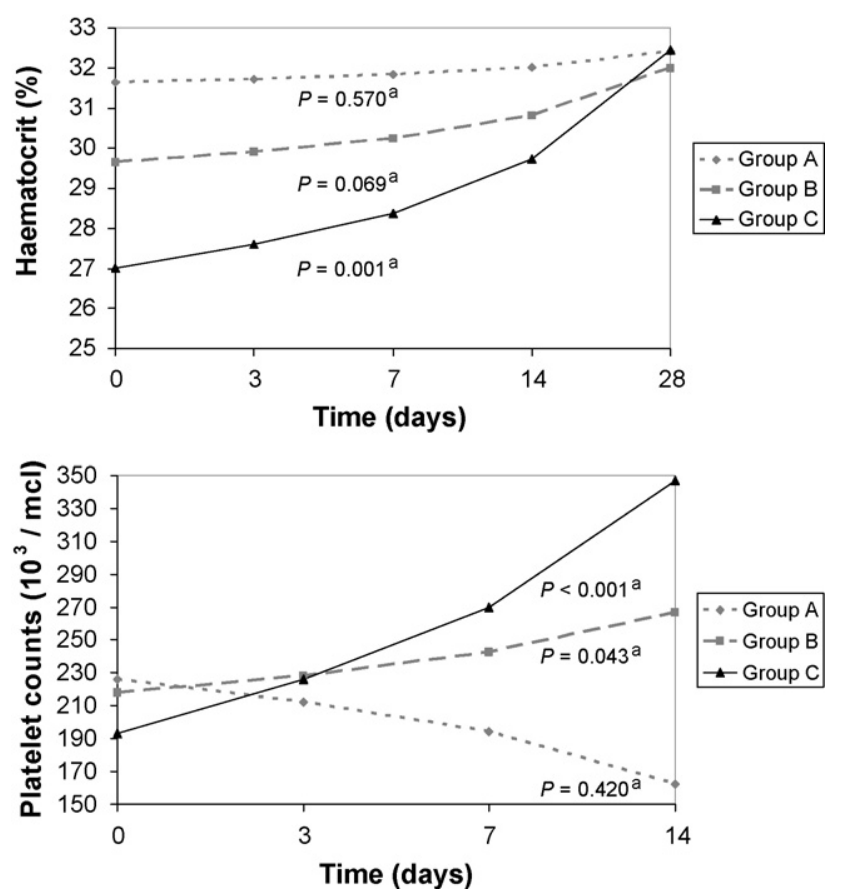

Figure 3 Change in average haematocrit (\%) and platelet counts stratified by treatment group in patients receiving Argemone mexicana decoction (haematocrit: $n=74$ on Day $0, n=43$ on Day 14 and $n=27$ on Day 28; platelets: $n=55$ on Day $0, n=44$ on Day 7 and $n=34$ on Day 14). a Statistical significance of slope. 
significant trend was observed in Group A $(P=0.420)$ (Figure 3 ). Increases in haematocrit and platelet counts towards normal levels are expected with successful treatment. There was no evidence of bone marrow toxicity at the doses given.

Twenty-four of the included patients had at least three results for biochemical parameters. There were no significant differences in creatinine between Day 0 and subsequent days, except in two patients who had an elevated creatinine at Day 0 , which returned to normal on subsequent tests. Seven patients (mainly in Group B) had a mild transient elevation of AST and/or ALT at Days 3 or 7 , which returned to normal by Day 28.

Thirteen patients had ECGs recorded. Significant ECG changes were only seen in two patients in Group C, one of whom had taken more than the prescribed dose. They developed corrected QT intervals $>500$ ms on Day 3 , returning to normal by Days 7-14. This was accompanied by flattening of the $T$ waves and appearance of $U$ waves. Testing of the stored serum samples for these patients revealed that their potassium levels were normal, so these changes are presumed to be a direct effect of the herbal remedy at high dose.

\section{Discussion}

\subsection{Statement of principal findings}

The decoction of $A$. mexicana used in this study appears to be effective for the treatment of uncomplicated malaria when taken for at least 7 days with two doses a day (Group B). Patients taking this dose had significantly better clinical results than those taking only one dose daily for 3 days (Group A). There appeared to be no advantage in taking the decoction four times a day for the first few days (Group C), and this dosage was associated with an increased risk of cardiac toxicity, as evidenced by QT prolongation seen in the ECGs of two patients.

These overall results must be interpreted in the context of the patient's age. Patients aged $<1$ year had poor results, possibly because young children often refused to drink an adequate quantity of the decoction and did not receive the preparation by other recommended routes such as washing. Furthermore, immunity to malaria is not well developed in this age group. In those aged 1-5 years, there was a very significant difference between Group A and Group B (25\% vs. $71 \%$ ACR at Day 14), suggesting that this is the age group where the treatment can make the biggest potential difference in outcome. Patients aged $>5$ years tended to have a good outcome, presumably because of a better developed immune response.

\subsection{Strengths and weaknesses of the study}

This study was not randomised, therefore confounding factors could play some part in the differences between groups. The temperature threshold for Group A was higher than for subsequent groups. However, significant differences remain even when adjusting for differences in baseline characteristics such as parasite count, splenomegaly or temperature.
There were insufficient numbers of patients in the groups aged $<1$ year and $>5$ years to be able to determine any effect of dosage in these groups. The decoction of $A$. mexicana was prepared by the traditional healer and there was no standardisation, so there could have been variations between batches.

\subsection{Strengths and weaknesses in relation to other studies, discussing important differences in results}

This is one of the larger cohort studies of a traditional herbal medicine for malaria and is unusual in including a majority of children $<5$ years, who are the main target group for malaria treatment (Willcox and Bodeker, 2004). In particular, the current 'improved traditional medicine' on the Malian national formulary, 'Malarial', was not evaluated in patients under the age of 5 years (Diallo et al., 2004). Although it is difficult to compare results between trials done with different populations and slightly different protocols, the reduction in parasitaemia at Day 14 appears to be better with $A$. mexicana than with 'Malarial'.

In several countries, Artemisia annua herbal tea has been investigated as a local herbal treatment for malaria, but no published trials of this have included children under the age of 11 years. The latest trial showed a parasite clearance rate of up to $77 \%$ at Day 7 (in patients aged $\geq 18$ years), but only $38 \%$ of patients remained free of parasites at Day 28 (Mueller et al., 2004). Unfortunately, clinical efficacy criteria were not used. Whilst $A$. annua is without doubt a very promising herbal antimalarial, it does not grow well in arid areas and trials of its cultivation in Mali gave disappointing results. In contrast, $A$. mexicana thrives in the arid Sahel climate, making it a very promising alternative in these areas.

Unlike newer antimalarials such as artemisinin combination therapies, the preparation used in this study did not produce complete clearance of parasites in most patients. However, in a setting where transmission is high, the relevance of complete parasite clearance is debatable. A study in Kampala, Uganda, suggested that asymptomatic parasitaemia increases the risk of subsequent clinical malaria (Njama-Meya et al., 2004), but this finding was not replicated in a study in Mali, where the epidemiology was more similar to that in our study (Bouvier et al., 1997). Even when clearance is achieved, re-infection will occur rapidly (Yeka et al., 2005). Indeed, in Bandiagara (central Mali), treatment of asymptomatic carriers with sulfadoxine/pyrimethamine to clear parasites did not result in a decreased incidence of clinical malaria over the whole season, but in an incidence lower in the first 4 weeks of follow-up, similar to the untreated group in Weeks 4-8, then more than double the untreated group in Weeks 9-12. The infection-free interval may have attenuated naturally acquired immunity (Coulibaly et al., 2002).

\subsection{Meaning of the study: possible explanations and implications for clinicians and policy-makers}

Effectiveness depends not only on efficacy but also on access, cost and rational drug use. In the context of remote areas where modern drugs are often lacking, unaffordable, misused or counterfeit (Basco, 2004; Dossou-Yovo et al., 
2001; Tumwesigire and Watson, 2002), it is entirely possible that $A$. mexicana could prove effective at a programmatic level. Further research is needed to test this hypothesis. In remote villages such as the setting for this study, $A$. mexicana is more accessible, more affordable and may be associated with better compliance than any modern drugs, including chloroquine. If further research confirms its effectiveness, $A$. mexicana could be proposed for malaria home treatment programmes in areas with difficulties of access.

\subsection{Unanswered questions and future research}

It is important to see whether the preparation of $A$. mexicana can be modified to increase its efficacy further. A variety of preparations of this plant are used in the Sikasso region of Mali (infusions, raw macerations, decoctions where the plant is boiled for only a few minutes). It is intended to test these in vitro in order to attempt to produce an improved formulation and to test other ways of administration for small children.

Bibliographic data indicated the presence of the alkaloid berberine, a well known antimalarial compound, in A. mexicana. Chromatographic and densitometric methods allowed us to measure approximately $20 \mathrm{mg} / \mathrm{l}$ of berberine in the village decoction (dosage confirmed later by HPLC). This amount of berberine is sufficient to explain the high in vitro activity displayed by $A$. mexicana extracts, but it is probably insufficient to explain the observed therapeutic effect of the decoction, since berberine is poorly absorbed orally. This suggests the presence of other active compounds, which may either act synergistically or enhance the oral bioavailability of berberine. Further analyses are currently in progress to address this question, not only because of potential pharmacological interest, but also to provide a chemical marker for quality control and selection in plant domestication.

Although there was no evidence of serious toxicity at dose ' $B$ ' of the decoction, further toxicity tests will be carried out on $A$. mexicana. QT prolongation was not specifically assessed in the animal toxicity studies, but the lack of lethality of even very high doses in the animal study suggests that when taken alone the decoction is safe. Argemone mexicana contains at least two alkaloids, protopine and allocryptopine, which are known to prolong the refractory period of cardiac myocytes and could account for the QT prolongation (McCawley, 1955). However, there were no observed cases of symptomatic cardiac toxicity in this study or in a subsequent larger study. There is a theoretical risk of cardiac arrhythmia in cases of extreme QT interval prolongation. In practice this is unlikely to happen with $A$. mexicana decoction alone, and it would only be a significant risk if patients were concurrently taking certain modern drugs that also prolong the QT interval (for example halofantrine). In remote villages, the use of modern drugs is unusual on a long-term basis and it would be very unlikely that a patient would take a modern drug at the same time as a herbal medicine.

\section{Conclusion}

Argemone mexicana decoction given twice daily for 7 days resulted in significantly more ACRs than a lower dose in the treatment of uncomplicated falciparum malaria. It led to an ACR at Day 14 in $73 \%$ of all patients and in $89 \%$ of those aged $>5$ years. There were no serious side effects.

Authors' contributions: MLW, BG, JF and DD designed the study protocol; MLW and OS carried out the clinical assessment, laboratory analyses and follow-up; all authors worked on analysis and interpretation of the data; MLW drafted the manuscript. All authors read and approved the final manuscript. MLW is guarantor of the paper.

Acknowledgements: The authors wish to thank the people and elders of Missidougou, in particular chief Tiemoko Bengaly, without whose open and enthusiastic collaboration this study would not have been possible. We are also very grateful to our fieldwork staff, Mr Diafara Berthé, Mr Yussuf Berthé, Mr Fagnan Sanogo and Dr Sanoussy Daffé, for their hard work in the field. We would like to thank the staff and doctoral students of the Département de Médecine Traditionnelle, the Malian health authorities, the Malaria Research and Training Centre, the National Malaria Control Programme, the WHO Mali Office, the PSDS office in Sikasso, Dr Sergio Giani, Prof. Bernard Burnand and Prof. André Rougemont for their invaluable advice in the planning and analysis of this study, as well as Reto Brun and the staff of the Swiss Tropical Institute.

Funding: Swiss Agency for Development and Cooperation (SDC).

Conflicts of interest: None declared.

Ethical approval: Ethics Committee of L'Institut National de Recherche en Santé Publique' (INRSP) of Mali.

\section{References}

Adjobimey, T., Edayé, I., Lagnika, L., Gbenou, J., Moudachirou, M., Sanni, A., 2004. Activités antiplasmodiales in vitro de quelques plantes antipaludiques de la pharmacopée béninoise. C. R. Chimie 7, 1023-1027.

Anonymous, 2005. Reversing the failures of Roll Back Malaria. Lancet $365,1439$.

Basco, L.K., 2004. Molecular epidemiology of malaria in Cameroon. XIX. Quality of antimalarial drugs used for self-medication. Am. J. Trop. Med. Hyg. 70, 245-250.

Bouvier, P., Rougemont, A., Breslow, N., Doumbo, O., Delley, V., Dicko, A., Diakite, M., Mauris, A., Robert, C.F., 1997. Seasonality and malaria in a west African village: does high parasite density predict fever incidence? Am. J. Epidemiol. 145, 850-857.

Coulibaly, D., Diallo, D.A., Thera, M.A., Dicko, A., Guindo, A.B., Kone, A.K., Cissoko, Y., Coulibaly, S., Djimde, A., Lyke, K., Doumbo, O.K., Plowe, C.V., 2002. Impact of preseason treatment on incidence of falciparum malaria and parasite density at a site for testing malaria vaccines in Bandiagara, Mali. Am. J. Trop. Med. Hyg. 67, 604-610.

Diallo, D., Maiga, A., Diakité, C., Willcox, M., 2004. 'Malarial5': development of an antimalarial phytomedicine in Mali, in: Willcox, M.L., Bodeker, G., Rasoanaivo, P. (Eds), Traditional Medicinal Plants and Malaria. CRC Press, Boca Raton, FL.

Diallo, D., Graz, B., Falquet, J., Traoré, A.K., Giani, S., Mounkoro, P.P., Berthé, A., Sacko, M., Diakité, C., 2006. Malaria treatment 
in remote areas of Mali: use of modern and traditional medicines, patient outcome. Trans. R. Soc. Trop. Med. Hyg. 100, 515-520.

Dossou-Yovo, J., Amalaman, K., Carnevale, P., 2001. Antimalarial guidelines and therapy practices of the population of Bouake Ivory Coast. Med. Trop. (Mars.) 61, 495-499 [in French].

Emmart, E.W., 1940. The Badianus Manuscript: an Aztec herbal of 1552. Johns Hopkins Press, Baltimore, MD.

Graz, B., Diallo, D., Falquet, J., Willcox, M., 2005. Screening of traditional herbal medicine: first, do a retrospective study, with correlation between diverse treatments used and reported patient outcome. J. Ethnopharmacol. 101, 338-339.

McCawley, E.L., 1955. Cardioactive alkaloids. in: Manske, E.H.F. (Ed.), The Alkaloids, Vol. 5. Academic Press, New York, NY, pp. 79-107.

Ministère de la Santé, des Personnes Agées et de la Solidarité, 1998. Formulaire Thérapeutique National. Mali Edition, Bamako, Mali.

Mueller, M.S., Runyambo, N., Wagner, I., Borrmann, S., Dietz, K., Heide, L., 2004. Randomized controlled trial of a traditional preparation of Artemisia annua L. (Annual Wormwood) in the treatment of malaria. Trans. R. Soc. Trop. Med. Hyg. 98, 318-321.

Nadkarni, A.K., 1976. Dr K.M. Nadkarni's Indian Materia Medica. Popular Prakashan, Bombay.

Njama-Meya, D., Kamya, M.R., Dorsey, G., 2004. Asymptomatic parasitaemia as a risk factor for symptomatic malaria in a cohort of Ugandan children. Trop. Med. Int. Health 9, 862-868.

Raudenbush, S.W., 2001. Comparing personal trajectories and drawing causal inferences from longitudinal data. Annu. Rev. Psychol. 52, 501-525.

Raudenbush, S.W., Bryk, A.S., 2002. Hierarchical Linear Models: applications and data analysis methods, second ed. Sage Publications, London, UK.

Raudenbush, S., Bryk, A., Cheong, Y.F., Congdon, R., 2001. SSI Scientific Software International Inc. http://www.ssicentral.com [accessed 2 May 2007].
Sarkar, S.N., 1948. Isolation from Argemone oil of dihydrosanguinarine and sanguinarine: toxicity of sanguinarine. Nature 162 , 265-266.

Sidibe, 0., 2006. Etude d'Argemone mexicana Linn. dans le traitement traditionnel du paludisme non compliqué dans le village de Missidougou, région de Sikasso, Mali. Doctoral thesis, Faculté de Médecine, Pharmacologie et Odonto-Stomatologie, University of Bamako, Mali.

Simonsen, H.T., Nordskjold, J.B., Smitt, U.W., Nyman, U., Palpu, P., Joshi, P., Varughese, G., 2001. In vitro screening of Indian medicinal plants for antiplasmodial activity. J. Ethnopharmacol. 74, 195-204.

Tandon, R.K., Singh, D.S., Arora, R.R., Lal, P., Tandon, B.N., 1975. Epidemic dropsy in New Delhi. Am. J. Clin. Nutr. 28, 883-887.

Tumwesigire, S., Watson, S., 2002. Health seeking behavior by families of children suspected to have malaria in Kabale, Uganda. Afr. Health Sci. 2, 94-98.

WHO, 1996. Assessment of Therapeutic Efficacy of Antimalarial Drugs. World Health Organization, Geneva, WHO/MAL/96.1077.

Willcox, M.L., Bodeker, G., 2004. Traditional herbal medicines for malaria. BMJ 329, 1156-1159.

Willcox, M.L., Olanrewaju, I., 2004. Guidelines for clinical studies on herbal antimalarials, in: Willcox, M.L., Bodeker, G., Rasoanaivo, P. (Eds), Traditional Medicinal Plants and Malaria. CRC Press, Boca Raton, FL.

Yeka, A., Banek, K., Bakyaita, N., Staedke, S.G., Kamya, M.R., Talisuna, A., Kironde, F., Nsobya, S.L., Kilian, A., Slater, M., Reingold, A., Rosenthal, P.J., Wabwire-Mangen, F., Dorsey, G., 2005. Artemisinin versus nonartemisinin combination therapy for uncomplicated malaria: randomized clinical trials from four sites in Uganda. PLoS Med 2, e190. 